\title{
Soil properties and the growth of wheat (Triticum aestivum L.) and maize (Zea mays L.) in response to reed (phragmites communis) biochar use in a salt-affected soil in the Yellow River Delta
}

\author{
Liang Xiao $^{\mathrm{a}, \mathrm{b}}$, Guodong Yuan ${ }^{\mathrm{a}, *}$, Lirong Feng ${ }^{\mathrm{b}}$, Dongxue Bi $^{\mathrm{b}}$, Jing Wei ${ }^{\mathrm{b}}$ \\ a Guangdong Provincial Key Laboratory of Environmental Health and Land Resource, Zhaoqing University, Zhaoqing, Guangdong, 526061, China \\ ${ }^{\mathrm{b}}$ Yantai Institute of Coastal Zone Research, Yantai, Shandong, 264003, China
}

\section{A R T I C L E I N F O}

\section{Keywords:}

K-rich biochar

saline soil

soil physical properties

SAR

nitrogen use efficiency

\begin{abstract}
A B S T R A C T
Soil salinity and its associated soil compaction and low fertility is a big problem for land management in the arid region or coastal zone. Here, a low-cost and potassium (K)-rich biochar of reed (phragmites communis) was demonstrated effective in alleviating the problem in wheat-maize rotation in the Yellow River Delta region. Adding the biochar at $0,3,6$, and $12 \mathrm{t} \mathrm{ha}^{-1}$ to a soil with a $2.8 \%$ salt content via rotary tillage with straw returning, with or without fertilizers, reduced soil bulk density (BD) and increased saturated hydraulic conductivity (Ks). At $12 \mathrm{t} \mathrm{ha}^{-1}$ dose and by wheat and maize harvests, respectively, biochar lowered soil BD by $9.1 \%$ and $14.5 \%$, increased $K$ s by $82.7 \%$ and $91.2 \%$, and reduced sodium adsorption ratio (SAR) by $64.9 \%$ and $92.8 \%$ in comparison with the control (CK). Further, in comparison with conventional fertilization (CF: $375 \mathrm{~kg} \mathrm{ha}^{-1}$ for each crop), biochar use ( 6 and $12 \mathrm{t} \mathrm{ha}^{-1}$ ), together with $75 \%$ of $\mathrm{CF}$, enhanced the nitrogen use efficiency (NUE) by $20.5 \%-31.4 \%$ for wheat and $15.9 \%-30.9 \%$ for maize. It raised the yields of wheat by $11.3 \%-17.1 \%$ and maize by $9.7 \%-14.8 \%$. By reducing $\mathrm{BD}$, increasing $\mathrm{Ks}$, and decreasing SAR, biochar alleviated soil compaction and salt stress and increased NUE and crop yields. This outcome suggests that the conversion of local bio-waste into biochar as a soil amendment is of agronomic and environmental benefits.
\end{abstract}

\section{Introduction}

The Yellow River Delta (YRD) is a fragile wetland ecosystem, characterized by high soil salinity and poor soil fertility (Luo et al., 2017). The excessive soluble salts and exchangeable sodium is a crucial constraint to crop growth (Zhang et al., 2015), resulting in low productivity (Wong et al. 2009), low biomass inputs to soil, soil compaction (Luo et al., 2017), poor nutrient retention, and low fertilizer use efficiency (Tedeschi et al., 2011). Besides, bio-waste disposal and over-fertilization are becoming new problems in the YRD (Wu et al., 2016; Shan et al., 2017). The use of straw returning and compost have been reported helpful in remediating salt-affected soil (Xie et al., 2017; Yang et al., 2018). Their side effects, such as nutrient deficiency, seedling growth restriction, and nematode problems (Gu et al., 2015), together with the high cost as a result of high usage and labour shortage (Sastre-Conde et al., 2015), make the large-scale use of compost and straw returning difficult in the YRD. Cost-effective and ecologically beneficial methods and technologies for alleviating soil salinity are much needed (Luo et al., 2017). Converting bio-waste into biochar is a promising means of alleviating the soil problems in the YRD.

Biochar is a carbon-rich material and typically produced by oxygenlimited pyrolysis of bio-waste (e.g., straw, branches, manure) (Lehmann et al., 2006). Biochar use as a soil amendment could improve the fertility and productivity of degraded soils (Alvarez-Campos et al., 2018; Al-Wabel et al., 2018; Yu et al., 2019). More specifically, its use in saline soil increased SOM content, soil fertility, field capacity (FC), and Ks (Chaganti et al., 2015), and decreased salt content, electric conductivity (EC), and sodium adsorption ratio (SAR) (Di Lonardo et al., 2017; Ali et al., 2017). These beneficial effects were attributed to the high carbon and nutrient contents (Ajayi et al., 2016), porous structure, large specific surface area (Blanco-Canqui 2017), and abundant functional groups of biochar (Nguyen et al., 2017). For example, biochar addition to saline soil improved soil porosity, FC and Ks, and accelerated salt leaching (Burrell et al., 2016; Obia et al., 2016); Sun et al. (2017) reported that biochar use at $5 \mathrm{~g} \mathrm{~kg}^{-1}$ reduced $\mathrm{NH}_{4}^{+}-\mathrm{N}$ and $\mathrm{NO}_{3}^{-}-\mathrm{N}$ leaching and $\mathrm{NH}_{3}$ volatilization, thus helped $\mathrm{N}$ retention in a coastal saline soil; Usman

\footnotetext{
* Corresponding author.

E-mail address: yuanguodong@zqu.edu.cn (G. Yuan).
} 
Table 1

The basic properties of biochar

\begin{tabular}{|c|c|c|c|c|c|c|c|c|c|c|c|c|c|c|}
\hline & 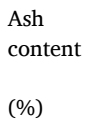 & 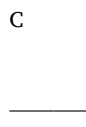 & - & $\mathrm{H}$ & - & $\ldots(\mathrm{m}$ & $\begin{array}{l}\text { Phenolic- } \\
\mathrm{OH} \\
\left.\mathrm{g}^{-1}\right)\end{array}$ & $\begin{array}{l}\text { Specific } \\
\text { surface } \\
\text { area } \\
\left(\mathrm{m}^{2} \mathrm{~g}^{-1}\right)\end{array}$ & $\mathrm{Na}^{+}$ & 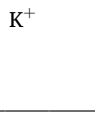 & (total, $\mathrm{mn}$ & $\mathrm{Mg}^{2+}$ & $\mathrm{Cl}^{-}$ & $\mathrm{SO}_{4}^{2-}$ \\
\hline Biochar & $\begin{array}{l}30.9 \pm \\
3.2\end{array}$ & $\begin{array}{l}43.5 \pm \\
0.0\end{array}$ & $\begin{array}{l}0.9 \pm \\
0.0\end{array}$ & $\begin{array}{l}2.2 \pm \\
0.0\end{array}$ & $\begin{array}{l}0.1 \pm \\
0.1\end{array}$ & $\begin{array}{l}1.1 \pm \\
0.0\end{array}$ & $0.5 \pm 0.0$ & $27.5 \pm 3.6$ & $\begin{array}{l}516.1 \\
\pm 9.9\end{array}$ & $\begin{array}{l}683.4 \pm \\
38.1\end{array}$ & $\begin{array}{l}227.7 \pm \\
15.8\end{array}$ & $\begin{array}{l}356.1 \pm \\
19.8\end{array}$ & $\begin{array}{l}859.4 \pm \\
47.2\end{array}$ & $\begin{array}{l}112.9 \\
\pm 8.6\end{array}$ \\
\hline
\end{tabular}

et al. (2016) and Zheng et al. (2018) concluded that biochar supplemented soil nutrients $(\mathrm{P}, \mathrm{K})$ and provided $\mathrm{Ca}^{2+}$ to replace $\mathrm{Na}^{+}$in soil, thus facilitating salt removal and nutrient retention (Yu et al., 2019). Despite the numerous reports on the potential agronomic and environmental benefits of biochar use as a soil amendment, field studies on the subject are inadequate in general and short in YRD (Saifullah et al., 2018; Al-Wabel et al., 2018). Most importantly, biochar for agricultural use has been restricted by its high cost (Vochozka et al., 2016; Saifullah et al., 2018).

This study aimed to use inexpensive biochar as a mediator for the interweaved problems of biowaste accumulation, soil salinity and compaction, and low soil productivity in YRD, with a hypothesis that biochar can lessen soil compaction and soil salinity, thus enhancing crop growth. To this end, low-cost biochar, produced in the filed from local bio-waste of reed, was added as a soil amendment with or without chemical fertilizers to a salt-affected soil in the YRD with wheat-maize rotation. Then, the physical and chemical properties of the soil at different crop-growing stages and the chemical compositions of wheat and maize grains were analyzed to assess the effect of biochar on soil properties and crop production. The outcomes from this research would help develop a management protocol of using biochar to remediate saltaffected soils.

\section{Materials and methods}

\subsection{Study area}

A field trial was conducted in an area of $67.5 \times 49.0 \mathrm{~m}^{2}$ in a farm in the Shenxiangou basin of Hekou District, Dongying City $\left(37^{\circ} 55.30^{\prime} \mathrm{N}\right.$, $118^{\circ} 48.88^{\prime} \mathrm{E}$ ). The farm is about 6500 ha in size, with a moderately salt content of $2.0 \%$ o- $3.0 \%$. Land reclamation started in the 1990 s by planting cotton. Since 2014 the farm has been in wheat-maize rotation, with land management practice of straw returning, base fertilization, and top dressing. The physical and chemical properties of the soil are shown in Table 1. This soil was chosen for a field trial because its moderate salt content is representative of farmland for wheat and maize production in the YRD. Soils with lower salt content $(<2.0 \%$ ) usually produce an acceptable yield without remediation, whereas soils with higher salt content $(>3.0 \%$ ) are not suitable for wheat and maize production before expensive remediation.

\subsection{Biochar, fertilizers, and crop seeds}

Bio-waste of reed (phragmites communis) was used to produce a lowcost biochar (\$24/ton) in the field via a fast aerobic carbonization process by fire-water coupled method (Xiao et al., 2019). The process had the dual features: aerobic combustion on the surface of reed and oxygen-limited pyrolysis inside. In brief, a pile of reed was ignited at multiple directions to produce dark red rods (with a measured surface temperature range of $274-282^{\circ} \mathrm{C}$ ). Water mist was then sprayed layer by layer on the rods to extinguish combustion and form biochar. Its properties were analyzed by established methods, as detailed in section 2.4.

As shown in Table 1, the biochar had moderate carbon content (43.5\%), SSA (27.5 $\left.\mathrm{m}^{2} \mathrm{~g}^{-1}\right)$, carboxyl group $\left(1.1 \mathrm{~mol} \mathrm{~kg}^{-1}\right)$, and phenolic hydroxyl group $\left(0.5 \mathrm{~mol} \mathrm{\textrm {kg } ^ { - 1 }}\right)$. The biochar had a much higher water- soluble $\mathrm{K}^{+}\left(407.1 \mathrm{mmol} \mathrm{kg}^{-1}\right)$ content than the soil $\left(3.3 \mathrm{mmol} \mathrm{kg}{ }^{-1}\right)$ (Table 2), its use as a soil amendment would alter the salt compositions of soil solution by releasing $\mathrm{K}^{+}$. On the other hand, its water-soluble $\mathrm{Na}^{+}$ $\left(333.8 \mathrm{mmol} \mathrm{kg}^{-1}\right)$ and $\mathrm{Cl}^{-}\left(330.2 \mathrm{mmol} \mathrm{kg}{ }^{-1}\right.$ ) suggest that cautions should be exercised to prevent secondary salinity from heavy use of the biochar in the absence of rain or irrigation.

Fertilizers were applied to the soil for each crop, as shown in Table 3. Base fertilization occurred a day before wheat and maize sowing (i.e., October $14^{\text {th }}, 2017$; June $19^{\text {th }}, 2018$ ), and topdressing was done a day before spring and autumn irrigations (March $20^{\text {th }}, 2018$; August $26^{\text {th }}$, 2018) with a hydrostatic pressure of $35 \mathrm{~cm}$ water each.

Wheat (Triticum aestivum L.) seeds of Jimai-22, with a 100-grain weight of $4.70 \mathrm{~g}$, were obtained from the Shandong Academy of Agricultural Sciences. Maize (Zea mays L.) seeds of Jishou-303, with a 100grain weight of $35.93 \mathrm{~g}$, were purchased from Dade Seeds Co. Ltd. of Beijing.

\subsection{Plot design, seed sowing, irrigation, and crop harvest}

Plots in the field trial were $10 \times 2 \mathrm{~m}^{2}$ with an isolation strip of $2 \mathrm{~m}$ long and $0.5 \mathrm{~m}$ wide. The biochar was crushed to less than $1 \mathrm{~mm}$, and then added once at $0,3,6$, and $12 \mathrm{tha}^{-1}$ (referred to as CK, T1, T2, and T3) to the topsoil $(0-20 \mathrm{~cm})$ by rotary tillage before wheat sowing. These doses were much smaller than those reported in the literature (Saifullah et al., 2018; Sun et al., 2019). As shown in Table 4, two levels of fertilization, namely conventional fertilization (CF, being $375 \mathrm{~kg} \mathrm{ha}^{-1}$ for each crop from a survey of local farmers) and reduced fertilization (being $75 \% \mathrm{CF}$ ), were used in the trial. Also included in the treatment were combinations of biochar use $\left(3,6\right.$, and $\left.12 \mathrm{t} \mathrm{ha}^{-1}\right)$ and $75 \% \mathrm{CF}$ (referred to as T4, T5, and T6). Treatment of CF without biochar was used as a benchmark for comparison of crop yield. Each treatment had 4 replications, and all plots in the field were randomized. Wheat seeds were sowed on October 15, 2017, at $188 \mathrm{~kg} \mathrm{ha}^{-1}$ with a furrow spacing of $15 \mathrm{~cm}$, and maize sowed on June 20,2018, at $25 \mathrm{~kg} \mathrm{ha}^{-1}$ with a furrow spacing of $35 \mathrm{~cm}$. Pesticides (imidacloprid and carbonfuran) were sprayed on May $10^{\text {th }}$ and July $20^{\text {th }}, 2018$, as routine practice for wheat and maize production. Wheat was harvested on June 10, 2018, and maize on October 5, 2018.

\subsection{Sample collection and analysis}

Soil samples were randomly collected from $0-15 \mathrm{~cm}$ layer of each plot following the S-shaped pattern in September 2017, June 2018, and October 2018 as the benchmark, wheat harvest, and maize harvest samples, respectively.

Wheat and maize harvests were carried out by whole-plot harvesting, and a thresher (5TF-450) was used to separate the grain from the straw. They were air-dried and weighed, randomly collected and mixed, ovendried at $85{ }^{\circ} \mathrm{C}$, crushed, and passed a 100 -mesh sieve for chemical analyses.

The biochar was analyzed for its ash content by heating at $800{ }^{\circ} \mathrm{C}$ for $4 \mathrm{~h}$ in a muffle furnace (Lu 1999), elemental compositions by an elemental analyzer (Vario Micro cube, Elementar, Germany), acidic functional groups by the titration method of the International Humic Substances Society (2019), and specific surface area (SSA) by $\mathrm{N}_{2}$ 
Table 2

Physical and chemical properties of the soil and biochar

\begin{tabular}{|c|c|c|c|c|c|c|c|c|c|c|c|c|c|c|c|}
\hline & $\begin{array}{l}\text { Bulk } \\
\text { density } \\
\text { ( } \mathrm{g} \\
\mathrm{cm}^{-3} \text { ) }\end{array}$ & $\begin{array}{l}\text { Capillary } \\
\text { porosity } \\
(\%)\end{array}$ & $\begin{array}{l}\text { Ks } \\
\left(\times 10^{-5}\right. \\
\left.\mathrm{cm} \mathrm{s}^{-1}\right)\end{array}$ & $\mathrm{pH}$ & $\begin{array}{l}\text { Salt } \\
\text { content } \\
(\% 0)\end{array}$ & $\begin{array}{l}\text { Organic } \\
\text { matter } \\
\left(\mathrm{g} \mathrm{kg}^{-1}\right)\end{array}$ & $\begin{array}{l}\mathrm{NH}_{4}^{+}- \\
\mathrm{N} \\
\end{array}$ & $\begin{array}{l}\mathrm{NO}_{3}^{-} \\
\mathrm{N}\end{array}$ & $\begin{array}{l}\text { Olsen- } \\
\mathrm{P} \\
\end{array}$ & 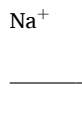 & $\begin{array}{l}\mathrm{K}^{+} \\
- \text {(wat }\end{array}$ & $\begin{array}{l}\mathrm{Ca}^{2+} \\
\text { soluble, }\end{array}$ & $\mathrm{Mg}^{2+}$ & $\mathrm{Cl}^{-}$ & $\mathrm{SO}_{4}^{2-}$ \\
\hline Soil & $\begin{array}{l}1.48 \pm \\
0.05\end{array}$ & $\begin{array}{l}29.5 \pm \\
2.50\end{array}$ & $\begin{array}{l}0.05 \pm \\
0.00\end{array}$ & $\begin{array}{l}8.2 \pm \\
0.2\end{array}$ & $\begin{array}{l}2.8 \pm \\
0.1\end{array}$ & $6.3 \pm 0.2$ & $\begin{array}{l}2.7 \pm \\
0.2\end{array}$ & $\begin{array}{l}27.1 \\
\pm 0.6\end{array}$ & $\begin{array}{l}0.8 \pm \\
0.0\end{array}$ & $\begin{array}{l}12.3 \pm \\
0.1\end{array}$ & $\begin{array}{l}3.3 \pm \\
0.1\end{array}$ & $\begin{array}{l}8.4 \pm \\
0.0\end{array}$ & $\begin{array}{l}3.5 \pm \\
0.1\end{array}$ & $\begin{array}{l}13.3 \pm \\
0.1\end{array}$ & $\begin{array}{l}2.4 \pm \\
0.1\end{array}$ \\
\hline Biochar & - & - & - & $\begin{array}{l}9.1 \pm \\
0.0\end{array}$ & - & - & $\begin{array}{l}21.6 \\
\pm 2.6\end{array}$ & $\begin{array}{l}0.9 \pm \\
0.1\end{array}$ & $\begin{array}{l}8.5 \pm \\
0.3\end{array}$ & $\begin{array}{l}333.8 \\
\pm 5.5\end{array}$ & $\begin{array}{r}407.1 \\
\pm 7.5\end{array}$ & $\begin{array}{l}16.5 \\
\pm 0.1\end{array}$ & $\begin{array}{l}31.3 \\
\pm 0.3\end{array}$ & $\begin{array}{l}330.2 \\
\pm 5.9\end{array}$ & $\begin{array}{l}55.4 \\
\pm 1.2\end{array}$ \\
\hline
\end{tabular}

Note: $K s$, Saturated hydraulic conductivity.

Table 3

The details of fertilizers and their applications

\begin{tabular}{|c|c|c|c|c|c|c|}
\hline Crop & Methods of application & Fertilizers & $\mathrm{N}$ content $(\%)$ & $\mathrm{P}$ content $(\%)$ & Conventional dose $\mathrm{kg} \mathrm{ha}^{-1}$ & Application dates \\
\hline \multirow{2}{*}{ Wheat } & Base & Urea-ammonium mixed nitrogen fertilizer & 29.96 & - & 187.5 & October $14^{\text {th }}, 2017$ \\
\hline & Topdressing & Slow-release fertilizer & 28.07 & 4.84 & 187.5 & March $20^{\text {th }}, 2018$ \\
\hline \multirow{2}{*}{ Mazie } & Base & Ammonium dihydrogen phosphate & 21.18 & 23.45 & 187.5 & June $19^{\text {th }}, 2018$ \\
\hline & Topdressing & Urea & 46.34 & - & 187.5 & August $26^{\text {th }}, 2018$ \\
\hline
\end{tabular}

Table 4

Experimental treatments of the plots

\begin{tabular}{|c|c|c|c|}
\hline Treatments & Detailed information & Treatments & Detailed information \\
\hline CK & 0 ton biochar per ha farmland $\left(0 \mathrm{t} \mathrm{ha}^{-1}\right)$ & $75 \% \mathrm{CF}$ & $281.25 \mathrm{~kg}$ fertilizers per ha farmland $\left(281.25 \mathrm{~kg} \mathrm{ha}^{-1}\right)$ \\
\hline $\mathrm{T} 1$ & 3 ton biochar per ha farmland $\left(3 \mathrm{t} \mathrm{ha}^{-1}\right)$ & $\mathrm{T} 4$ & $3 \mathrm{t} \mathrm{ha}^{-1}$ biochar and $281.25 \mathrm{~kg} \mathrm{ha}^{-1}$ fertilizers \\
\hline $\mathrm{T} 2$ & 6 ton biochar per ha farmland $\left(6 \mathrm{t} \mathrm{ha}^{-1}\right)$ & $\mathrm{T} 5$ & $6 \mathrm{t} \mathrm{ha}^{-1}$ biochar and $281.25 \mathrm{~kg} \mathrm{ha}^{-1}$ fertilizers \\
\hline $\mathrm{T} 3$ & 12 ton biochar per ha farmland $\left(12 \mathrm{t} \mathrm{ha}^{-1}\right)$ & T6 & $12 \mathrm{t} \mathrm{ha}^{-1}$ biochar and $281.25 \mathrm{~kg} \mathrm{ha}^{-1}$ fertilizers \\
\hline $\mathrm{CF}$ & $375 \mathrm{~kg}$ fertilizers per ha farmland (375 $\mathrm{kg} \mathrm{ha}^{-1}$ ) & - & - \\
\hline
\end{tabular}

sorption at $77 \mathrm{~K}$ using a Quantachrome Autosorb-iQ analyzer and applying the Brunauer-Emmett-Teller (BET) equation. The biochar was digested by $\mathrm{HNO}_{3}-\mathrm{HF}-\mathrm{H}_{2} \mathrm{O}_{2}$ for the analysis of total ions $\left(\mathrm{K}^{+}, \mathrm{Na}^{+}, \mathrm{Ca}^{2+}\right.$, $\mathrm{Mg}^{2+}, \mathrm{Cl}^{-}, \mathrm{SO}_{4}^{2-}$ ) by ion chromatography (ICS3000, Dionex) (Lu 1999).

Soil samples were determined for bulk density by the cutting ring method (Lu 1999), saturated hydraulic conductivity (Ks) by constant head test (Shao et al., 2006), salt content by weighing method (Bao 2000), and organic matter content by the wet oxidation method of $\mathrm{K}_{2} \mathrm{Cr}_{2} \mathrm{O}_{7}-\mathrm{H}_{2} \mathrm{SO}_{4}$ (Lu 1999).

Extracts (solutions) of biochar and soil samples were obtained by adding biochar or soil samples to $\mathrm{CO}_{2}$-free-deionized water at 1:5 ratio, shaking at $160 \mathrm{r} \mathrm{min}^{-1}$ for $24 \mathrm{~h}$, centrifuging at $3500 \mathrm{rpm}$ for $15 \mathrm{~min}$, and filtering through $0.45 \mu \mathrm{m}$ membrane for the measurement of $\mathrm{pH}$ by a $\mathrm{pH}$ meter (Five Easy Plus, METTLER TOLEDO), electrical conductivity (EC) by a conductivity meter (DDS-11A), and ion concentrations by ion chromatography (ICS3000, Dionex) (Bao 2000). The $\mathrm{NH}_{4}^{+}-\mathrm{N}$ and $\mathrm{NO}_{3}^{-}-\mathrm{N}$ of biochar and soil samples were extracted by $1 \mathrm{~mol} \mathrm{~L}^{-1} \mathrm{KCl}$. Olsen-P was extracted by $0.5 \mathrm{~mol} \mathrm{~L}^{-1} \mathrm{NaHCO}_{3}$ at a ratio of $1: 5(\mathrm{w} / \mathrm{v}$, shaking at $160 \mathrm{r}$ $\mathrm{min}^{-1}$ for $2 \mathrm{~h}$ ) and measured with a continuous flow analytical system (AutoAnalyzer III, Seal) (Lu 1999).

The fertilizers were digested by $\mathrm{H}_{2} \mathrm{SO}_{4}$ and then determined for total $\mathrm{N}$ contents by titration with $\mathrm{NaOH}$, and total $\mathrm{P}$ was measured by phosphorus molybdic acid quinoline weight method (Lu 1999).

Wheat and maize grain samples were digested by $\mathrm{HNO}_{3}-\mathrm{HF}-\mathrm{H}_{2} \mathrm{O}_{2}$ for the analysis of total $\mathrm{Na}, \mathrm{K}, \mathrm{Ca}$, and $\mathrm{Mg}$ by ICP-MS (Elan DRC II, PerkinElmer) (Lu 1999). The samples were digested by $\mathrm{H}_{2} \mathrm{SO}_{4}-\mathrm{H}_{2} \mathrm{O}_{2}$ for the determination of total $\mathrm{N}$ and $\mathrm{P}$ by Nessler's reagent and vanadium molybdate methods (Lu 1999).

\subsection{Data processing}

Two indexes were used to evaluate the effects of individual (i.e., biochar) and conjunctive (biochar $+75 \% \mathrm{CF}$ ) effects on alleviating soil salinity. Sodium adsorption ratio (SAR) was calculated by Eq. (1) (Lesch and Suarez 2009), and Chloride/ $\sqrt{ }$ sulfate ratio was calculated by Eq. (2) (Wang et al., 2018).

$$
\begin{aligned}
& \text { SAR }=\frac{\left[\mathrm{Na}^{+}\right]}{\sqrt{\left[\mathrm{Ca}^{2+}+\mathrm{Mg}^{2+}\right]}} \\
& \text { Chloride } / \sqrt{\text { Sulfate }}=\frac{\left[\mathrm{Cl}^{-}\right]}{\sqrt{\left[\mathrm{SO}_{4}^{2-}\right]}}
\end{aligned}
$$

where $\left[\mathrm{Na}^{+}\right],\left[\mathrm{Ca}^{2+}\right],\left[\mathrm{Mg}^{2+}\right],\left[\mathrm{Cl}^{-}\right]$and $\left[\mathrm{SO}_{4}^{2-}\right]\left(\mathrm{mmol} \mathrm{L}^{-1}\right)$ are the concentrations of the ions in soil solution.

SAR indicates the relative abundance of $\mathrm{Na}^{+}, \mathrm{Ca}^{2+}$, and $\mathrm{Mg}^{2+}$ in soil solution. As $\mathrm{Na}^{+}$is more harmful to crops than $\mathrm{Mg}^{2+}$ and $\mathrm{Ca}^{2+}$, the smaller the SAR is, the weaker the salt stress would be (Shaygan et al., 2017). In analogy to SAR, the ratio of chloride/ $\sqrt{ }$ sulfate indicates the relative concentration of $\mathrm{Cl}^{-}$and $\mathrm{SO}_{4}^{2-}$. As $\mathrm{Cl}^{-}$is much more toxic to plants than $\mathrm{SO}_{4}^{2-}$, a smaller ratio would suggest less salt stress.

$\mathrm{K} / \mathrm{Na}$ ratio in grains (Lin et al., 2015), indicates the ability of a crop to preferably take up $\mathrm{K}^{+}$rather than passively absorb toxic $\mathrm{Na}^{+}$.

Nitrogen and phosphorus use efficiency (NUE, PUE), calculated by Eq. (3), indicates the nutrient retention in soil and nutrient supply for wheat and maize growth (Lu et al., 2019).

NUE or PUE $=[($ total $\mathrm{N}$ or $\mathrm{P}$ of the crops in the plot where fertilizers were applied - total $\mathrm{N}$ or $\mathrm{P}$ of the crops in the plot of control) / the amount of $\mathrm{N}$ or $\mathrm{P}$ in fertilizers $\times 100 \%$ ] (3)

Excel 2013, SPSS 16.0, and Origin 8.0 were used for data management, statistical analysis, and figure drawing. One-way ANOVA was performed for statistical significance analysis (Duncan's test, $p<0.05$ ). 

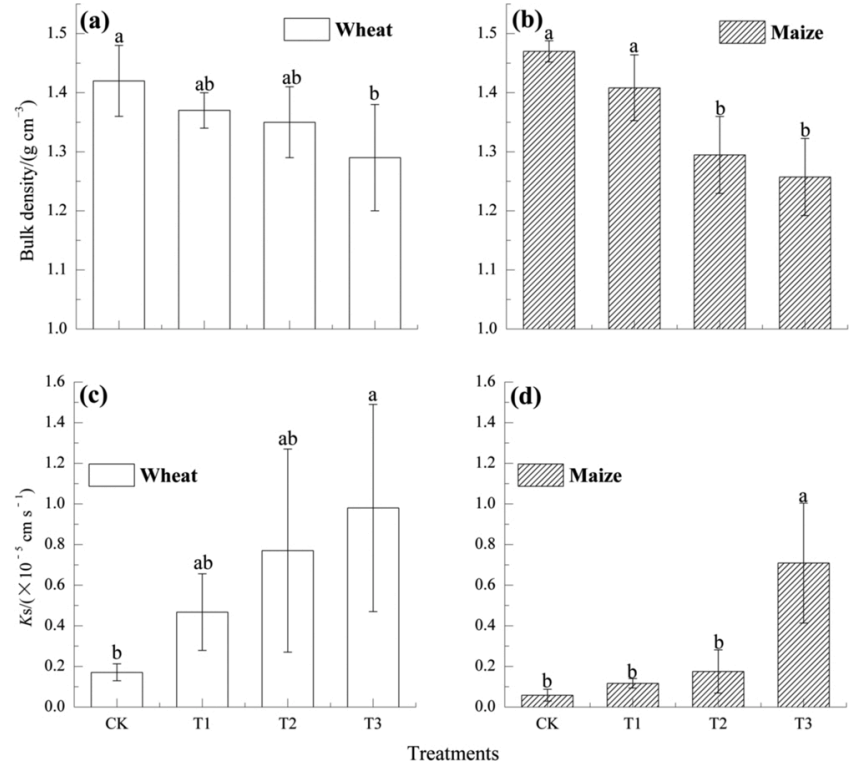

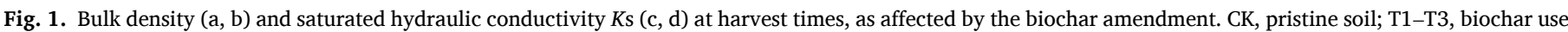
at 3, 6, $12 \mathrm{t} \mathrm{ha}^{-1}$. Different lower-case letters indicate significant differences between.

\section{Results}

\subsection{Response of soil physical properties to biochar use}

Use of the biochar reduced soil BD by $3.52 \%$ (T1), $4.93 \%$ (T2), and 9.15\% (T3) over the control (CK) at wheat harvest time, but only $12 \mathrm{t}$ $\mathrm{ha}^{-1}$ biochar dose (T3) showed a significant difference (Fig. 1a). Likewise, 6 and $12 \mathrm{t} \mathrm{ha}^{-1}$ biochar doses significantly reduced soil $\mathrm{BD}$ at maize harvest by $12.3 \%$ and $14.5 \%$ over the CK, respectively (Fig. 1b). In agreement with $\mathrm{BD}$ reduction, biochar application increased Ks. However, only the $12 \mathrm{t} \mathrm{ha}^{-1}$ biochar dose showed a statistically significant difference (Fig. 1c, d) at wheat and maize harvests from the CK. The decrease in $\mathrm{BD}$ and increase in $\mathrm{Ks}$ by biochar addition indicated the biochar amendment lessened the compaction, which in turn would affect salt and nutrient leaching.

\subsection{Response of soil chemical properties to biochar use}

As shown in Table 5, the concentrations of $\mathrm{Na}^{+}, \mathrm{K}^{+}, \mathrm{Ca}^{2+}, \mathrm{Mg}^{2+}, \mathrm{Cl}^{-}$, and $\mathrm{SO}_{4}^{2-}$ in soil solution were all significantly reduced by biochar treatments (T1-T3) over the $\mathrm{CK}$ at wheat and maize harvests. Compared with the $75 \% \mathrm{CF}$, the conjunctive effects of biochar and reduced fertilization treatments (T4-T6) on $\mathrm{Na}^{+}, \mathrm{K}^{+}, \mathrm{Ca}^{2+}$, and $\mathrm{Mg}^{2+}$ concentrations followed the same trend as biochar alone at wheat harvest time. Though ion concentrations of T4-T6 at maize harvest were higher than those of $75 \% \mathrm{CF}$, they were lower than CK. In other words, biochar alone (3-12 t $\mathrm{ha}^{-1}$ ) or in combination with $75 \% \mathrm{CF}$ reduced soluble salt concentrations, thus alleviating salt stress on the crops. Besides, biochar use $(12 \mathrm{t}$

Table 5

Concentrations of ions in soil extracts and soil $\mathrm{pH}$ at wheat and maize harvest

\begin{tabular}{|c|c|c|c|c|c|c|c|c|c|c|c|c|c|c|}
\hline \multirow[b]{2}{*}{ Treatments } & \multicolumn{2}{|l|}{$\mathrm{Na}^{+}$} & \multicolumn{2}{|l|}{$\mathrm{K}^{+}$} & \multicolumn{2}{|l|}{$\mathrm{Ca}^{2+}$} & \multicolumn{2}{|l|}{$\mathrm{Mg}^{2+}$} & \multicolumn{2}{|l|}{$\mathrm{Cl}^{-}$} & \multicolumn{2}{|l|}{$\mathrm{SO}_{4}^{2-}$} & \multicolumn{2}{|l|}{$\mathrm{pH}$} \\
\hline & $\begin{array}{l}\text { Wheat } \\
\text { harvest }\end{array}$ & $\begin{array}{l}\text { Maize } \\
\text { harvest }\end{array}$ & $\begin{array}{l}\text { Wheat } \\
\text { harvest }\end{array}$ & $\begin{array}{l}\text { Maize } \\
\text { harvest }\end{array}$ & $\begin{array}{l}\text { Wheat } \\
\text { harvest }\end{array}$ & $\begin{array}{l}\text { Maize } \\
\text { harvest }\end{array}$ & $\begin{array}{l}\text { Wheat } \\
\text { harvest } \\
\text { mmol L }^{-1} \text { ) }\end{array}$ & $\begin{array}{l}\text { Maize } \\
\text { harvest }\end{array}$ & $\begin{array}{l}\text { Wheat } \\
\text { harvest }\end{array}$ & $\begin{array}{l}\text { Maize } \\
\text { harvest }\end{array}$ & $\begin{array}{l}\text { Wheat } \\
\text { harvest }\end{array}$ & $\begin{array}{l}\text { Maize } \\
\text { harvest }\end{array}$ & $\begin{array}{l}\text { Wheat } \\
\text { harvest }\end{array}$ & $\begin{array}{l}\text { Maize } \\
\text { harvest }\end{array}$ \\
\hline CK & $\begin{array}{l}1.29 \pm \\
0.08 \mathrm{a}\end{array}$ & $\begin{array}{l}5.40 \pm \\
0.14 \mathrm{a}\end{array}$ & $\begin{array}{l}0.35 \pm \\
0.02 \mathrm{a}\end{array}$ & $\begin{array}{l}0.58 \pm \\
0.01 \mathrm{a}\end{array}$ & $\begin{array}{l}0.99 \pm \\
0.03 a\end{array}$ & $\begin{array}{l}1.41 \pm \\
0.03 \mathrm{a}\end{array}$ & $\begin{array}{l}0.35 \pm \\
0.02 \mathrm{a}\end{array}$ & $\begin{array}{l}0.90 \pm \\
0.02 \mathrm{a}\end{array}$ & $\begin{array}{l}1.65 \pm \\
0.01 \mathrm{a}\end{array}$ & $\begin{array}{l}5.87 \pm \\
0.16 \mathrm{a}\end{array}$ & $\begin{array}{l}0.49 \pm \\
0.17 \mathrm{a}\end{array}$ & $\begin{array}{l}0.90 \pm \\
0.02 \mathrm{a}\end{array}$ & $\begin{array}{l}7.87 \pm \\
0.01 \mathrm{a}\end{array}$ & $\begin{array}{l}8.07 \pm \\
0.03 a\end{array}$ \\
\hline $\mathrm{T} 1$ & $\begin{array}{l}0.07 \pm \\
0.00 \mathrm{~b}\end{array}$ & $\begin{array}{l}0.23 \pm \\
0.01 b\end{array}$ & $\begin{array}{l}0.04 \pm \\
0.00 \mathrm{~b}\end{array}$ & $\begin{array}{l}0.30 \pm \\
0.00 \mathrm{~b}\end{array}$ & $\begin{array}{l}0.01 \pm \\
0.00 \mathrm{~b}\end{array}$ & $\begin{array}{l}0.70 \pm \\
0.03 \mathrm{bc}\end{array}$ & $\begin{array}{l}0.02 \pm \\
0.00 \mathrm{~b}\end{array}$ & $\begin{array}{l}0.20 \pm \\
0.00 \mathrm{~b}\end{array}$ & $\begin{array}{l}0.25 \pm \\
0.00 c\end{array}$ & $\begin{array}{l}0.15 \pm \\
0.00 \mathrm{~b}\end{array}$ & $\begin{array}{l}0.07 \pm \\
0.01 b\end{array}$ & $\begin{array}{l}0.05 \pm \\
0.00 \mathrm{~b}\end{array}$ & $\begin{array}{l}7.76 \pm \\
0.05 \mathrm{ab}\end{array}$ & $\begin{array}{l}7.94 \pm \\
0.03 \mathrm{~b}\end{array}$ \\
\hline $\mathrm{T} 2$ & $\begin{array}{l}0.07 \pm \\
0.00 \mathrm{~b}\end{array}$ & $\begin{array}{l}0.23 \pm \\
0.01 b\end{array}$ & $\begin{array}{l}0.03 \pm \\
0.00 \mathrm{~b}\end{array}$ & $\begin{array}{l}0.26 \pm \\
0.00 c\end{array}$ & $\begin{array}{l}0.01 \pm \\
0.00 \mathrm{~b}\end{array}$ & $\begin{array}{l}0.64 \pm \\
0.01 c\end{array}$ & $\begin{array}{l}0.02 \pm \\
0.00 \mathrm{~b}\end{array}$ & $\begin{array}{l}0.19 \pm \\
0.01 b\end{array}$ & $\begin{array}{l}0.31 \pm \\
0.00 \mathrm{~b}\end{array}$ & $\begin{array}{l}0.11 \pm \\
0.00 \mathrm{~b}\end{array}$ & $\begin{array}{l}0.06 \pm \\
0.00 \mathrm{~b}\end{array}$ & $\begin{array}{l}0.03 \pm \\
0.00 \mathrm{~b}\end{array}$ & $\begin{array}{l}7.48 \pm \\
0.20 \mathrm{~b}\end{array}$ & $\begin{array}{l}8.05 \pm \\
0.01 a\end{array}$ \\
\hline T3 & $\begin{array}{l}0.07 \pm \\
0.01 b\end{array}$ & $\begin{array}{l}0.26 \pm \\
0.00 \mathrm{~b}\end{array}$ & $\begin{array}{l}0.04 \pm \\
0.00 \mathrm{~b}\end{array}$ & $\begin{array}{l}0.25 \pm \\
0.00 c\end{array}$ & $\begin{array}{l}0.01 \pm \\
0.00 \mathrm{~b}\end{array}$ & $\begin{array}{l}0.72 \pm \\
0.01 b\end{array}$ & $\begin{array}{l}0.02 \pm \\
0.00 \mathrm{~b}\end{array}$ & $\begin{array}{l}0.25 \pm \\
0.00 \mathrm{~b}\end{array}$ & $\begin{array}{l}0.15 \pm \\
0.00 \mathrm{~d}\end{array}$ & $\begin{array}{l}0.18 \pm \\
0.01 b\end{array}$ & $\begin{array}{l}0.06 \pm \\
0.00 \mathrm{~b}\end{array}$ & $\begin{array}{l}0.04 \pm \\
0.00 \mathrm{~b}\end{array}$ & $\begin{array}{l}7.61 \pm \\
0.14 \mathrm{ab}\end{array}$ & $\begin{array}{l}7.94 \pm \\
0.03 \mathrm{~b}\end{array}$ \\
\hline $\mathrm{CF}$ & $\begin{array}{l}0.88 \pm \\
0.01\end{array}$ & $\begin{array}{l}0.20 \pm \\
0.00\end{array}$ & $\begin{array}{l}0.35 \pm \\
0.01\end{array}$ & $\begin{array}{l}0.23 \pm \\
0.02\end{array}$ & $\begin{array}{l}0.58 \pm \\
0.04\end{array}$ & $\begin{array}{l}0.64 \pm \\
0.02\end{array}$ & $\begin{array}{l}0.23 \pm \\
0.02\end{array}$ & $\begin{array}{l}0.19 \pm \\
0.01\end{array}$ & $\begin{array}{l}0.23 \pm \\
0.00\end{array}$ & $\begin{array}{l}0.14 \pm \\
0.01\end{array}$ & $\begin{array}{l}0.09 \pm \\
0.00\end{array}$ & $\begin{array}{l}0.05 \pm \\
0.00\end{array}$ & $\begin{array}{l}7.53 \pm \\
0.13\end{array}$ & $\begin{array}{l}8.01 \pm \\
0.01\end{array}$ \\
\hline $75 \%$ CF & $\begin{array}{l}0.62 \pm \\
0.02 \mathrm{a}\end{array}$ & $\begin{array}{l}0.25 \pm \\
0.01 \mathrm{c}\end{array}$ & $\begin{array}{l}0.37 \pm \\
0.01 \mathrm{a}\end{array}$ & $\begin{array}{l}0.24 \pm \\
0.01 b\end{array}$ & $\begin{array}{l}0.63 \pm \\
0.02 \mathrm{a}\end{array}$ & $\begin{array}{l}0.72 \pm \\
0.05 c\end{array}$ & $\begin{array}{l}0.21 \pm \\
0.00 \mathrm{a}\end{array}$ & $\begin{array}{l}0.23 \pm \\
.0 .01 \mathrm{~b}\end{array}$ & $\begin{array}{l}0.14 \pm \\
0.00 c\end{array}$ & $\begin{array}{l}0.13 \pm \\
0.03 \mathrm{~d}\end{array}$ & $\begin{array}{l}0.06 \pm \\
0.01 c\end{array}$ & $\begin{array}{l}0.02 \pm \\
0.00 \mathrm{~d}\end{array}$ & $\begin{array}{l}7.65 \pm \\
0.06 \mathrm{a}\end{array}$ & $\begin{array}{l}7.93 \pm \\
0.01 \mathrm{a}\end{array}$ \\
\hline $\mathrm{T} 4$ & $\begin{array}{l}0.07 \pm \\
0.00 \mathrm{~b}\end{array}$ & $\begin{array}{l}0.50 \pm \\
0.01 b\end{array}$ & $\begin{array}{l}0.03 \pm \\
0.00 \mathrm{~b}\end{array}$ & $\begin{array}{l}0.20 \pm \\
0.00 c\end{array}$ & $\begin{array}{l}0.01 \pm \\
0.00 \mathrm{~b}\end{array}$ & $\begin{array}{l}0.84 \pm \\
0.03 b\end{array}$ & $\begin{array}{l}0.02 \pm \\
0.00 \mathrm{~b}\end{array}$ & $\begin{array}{l}0.23 \pm \\
0.00 \mathrm{~b}\end{array}$ & $\begin{array}{l}0.36 \pm \\
0.01 b\end{array}$ & $\begin{array}{l}0.82 \pm \\
0.02 b\end{array}$ & $\begin{array}{l}0.15 \pm \\
0.01 b\end{array}$ & $\begin{array}{l}0.14 \pm \\
0.00 \mathrm{~b}\end{array}$ & $\begin{array}{l}7.58 \pm \\
0.02 \mathrm{a}\end{array}$ & $\begin{array}{l}7.84 \pm \\
0.01 \mathrm{~b}\end{array}$ \\
\hline T5 & $\begin{array}{l}0.07 \pm \\
0.00 \mathrm{~b}\end{array}$ & $\begin{array}{l}0.27 \pm \\
0.01 c\end{array}$ & $\begin{array}{l}0.04 \pm \\
0.01 b\end{array}$ & $\begin{array}{l}0.32 \pm \\
0.00 \mathrm{a}\end{array}$ & $\begin{array}{l}0.02 \pm \\
0.01 b\end{array}$ & $\begin{array}{l}0.63 \pm \\
0.01 \mathrm{c}\end{array}$ & $\begin{array}{l}0.02 \pm \\
0.00 \mathrm{~b}\end{array}$ & $\begin{array}{l}0.18 \pm \\
000 c\end{array}$ & $\begin{array}{l}0.14 \pm \\
0.00 c\end{array}$ & $\begin{array}{l}0.21 \pm \\
0.00 c\end{array}$ & $\begin{array}{l}0.05 \pm \\
0.00 c\end{array}$ & $\begin{array}{l}0.04 \pm \\
0.00 c\end{array}$ & $\begin{array}{l}7.42 \pm \\
0.18 \mathrm{a}\end{array}$ & $\begin{array}{l}7.94 \pm \\
0.00 \mathrm{a}\end{array}$ \\
\hline T6 & $\begin{array}{l}0.07 \pm \\
0.00 \mathrm{~b}\end{array}$ & $\begin{array}{l}1.89 \pm \\
0.01 \mathrm{a}\end{array}$ & $\begin{array}{l}0.04 \pm \\
0.00 \mathrm{~b}\end{array}$ & $\begin{array}{l}0.32 \pm \\
0.01 \mathrm{a}\end{array}$ & $\begin{array}{l}0.01 \pm \\
0.00 \mathrm{~b}\end{array}$ & $\begin{array}{l}1.21 \pm \\
0.03 \mathrm{a}\end{array}$ & $\begin{array}{l}0.02 \pm \\
0.00 \mathrm{~b}\end{array}$ & $\begin{array}{l}0.48 \pm \\
0.01 \mathrm{a}\end{array}$ & $\begin{array}{l}0.60 \pm \\
0.00 \mathrm{a}\end{array}$ & $\begin{array}{l}1.90 \pm \\
0.01 \mathrm{a}\end{array}$ & $\begin{array}{l}0.31 \pm \\
0.01 \mathrm{a}\end{array}$ & $\begin{array}{l}0.51 \pm \\
0.01 \mathrm{a}\end{array}$ & $\begin{array}{l}7.61 \pm \\
0.20 \mathrm{a}\end{array}$ & $\begin{array}{l}7.77 \pm \\
0.00 \mathrm{c}\end{array}$ \\
\hline
\end{tabular}

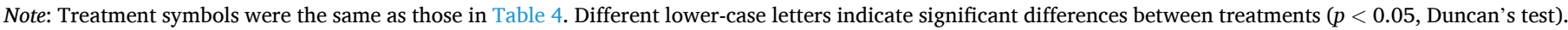



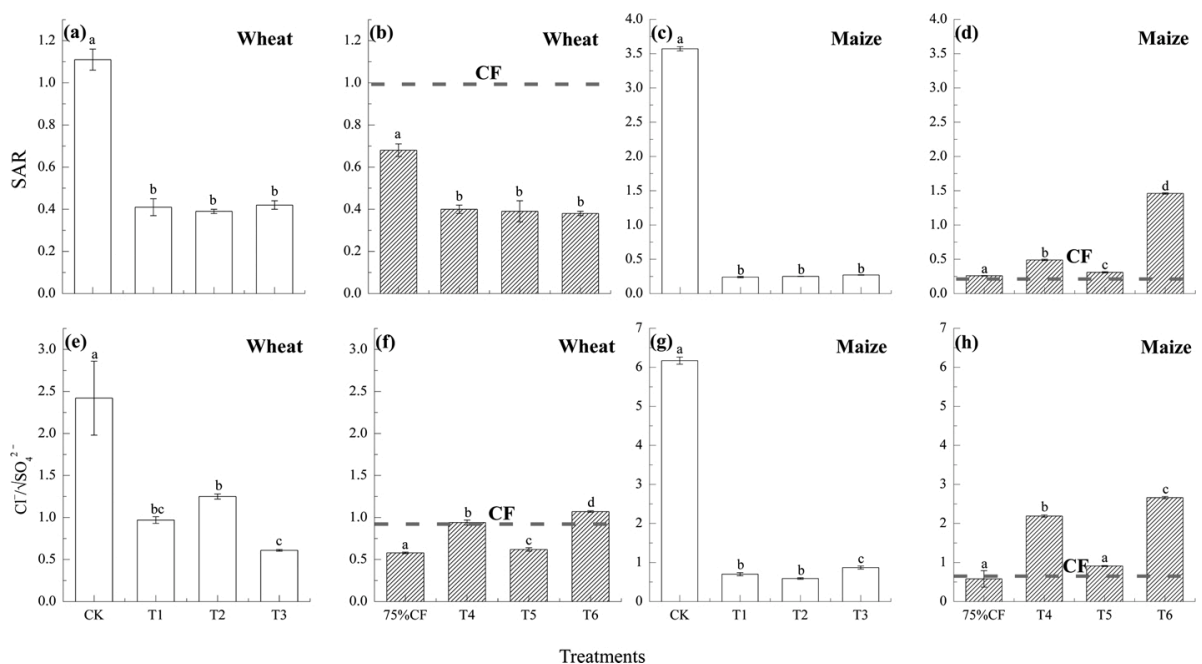

Fig. 2. Sodium adsorption ratio (a-d) and $\mathrm{Cl}^{-} / \sqrt{ } \mathrm{SO}_{4}^{2-}(\mathrm{e}-\mathrm{h}$ ) at harvest times. $\mathrm{CK}$ and $\mathrm{T} 1-\mathrm{T} 3$ were the same as those in Fig. 1. CF (dotted line) was the conventional fertilization ( $375 \mathrm{~kg} \mathrm{ha}^{-1}$ ); 75\% CF was 75\% of the CF. T4-T6 were treatments of biochar at 3, 6, $12 \mathrm{t} \mathrm{ha}^{-1}$ together with $75 \% \mathrm{CF}$. Different lower-case letters indicate significant differences between treatments ( $p<0.05$, Duncan's test).

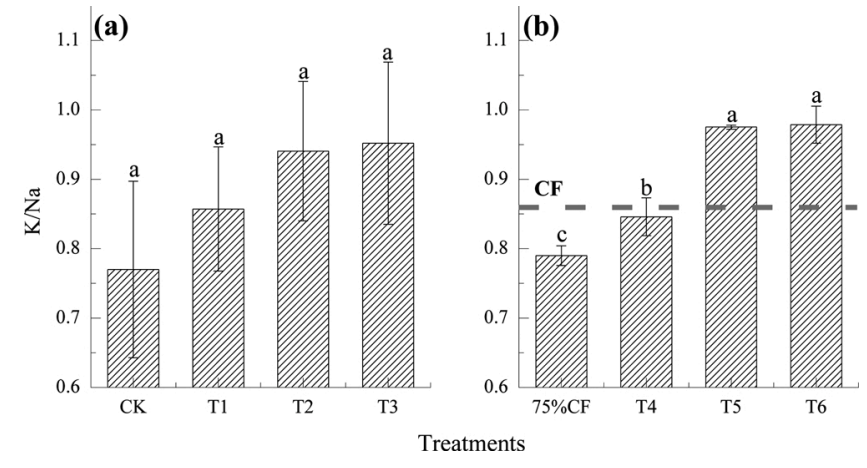

Fig. 3. K/Na ratio in wheat grain as affected by biochar (a) and co-application of biochar and fertilizers (b). The treatments were the same as those in Fig. 2. Different lower-case letters indicate significant differences between treatments $(p<0.05$, Duncan's test).

$\mathrm{ha}^{-1}$ ) alone or in combination with $75 \% \mathrm{CF}$ reduced soil $\mathrm{pH}$ at wheat and maize harvest times.

Fig. 2 shows changes in soil solution compositions. Biochar addition at $3-12 \mathrm{t} \mathrm{ha}^{-1}$ (T1-T3) significantly decreased SAR by $62.16 \%-64.86 \%$ and $\mathrm{Cl}^{-} / \sqrt{ } \mathrm{SO}_{4}^{2-}$ by $48.35 \%-74.79 \%$ over $\mathrm{CK}$ at wheat harvest time (Fig. 2a, e). Similar effects on SAR and $\mathrm{Cl}^{-} / \sqrt{ } \mathrm{SO}_{4}^{2-}$ were obtained at maize harvest (Fig. 2c, g). Because biochar alone reduced the relative abundance of the highly toxic $\mathrm{Na}^{+}$and $\mathrm{Cl}^{-}$, its use as a soil amendment alleviated soil salinity not only by lowering salt concentrations but also by producing less toxic salt compositions for crops.

The conjunctive effect of biochar and fertilizer use (T4-T6) on SAR and $\mathrm{Cl}^{-} / \sqrt{\mathrm{SO}_{4}^{2-}}$ were complex. SAR was reduced at wheat harvest (Fig. 2b) but increased at maize harvest (Fig. 2d) over $75 \%$ CF. This inconsistency was probably due to the more complex dry-wet alternations during the maize growth than the wheat season, resulting in the circular upward movement of salty groundwater in the soil profile and salt accumulation (dominated by $\mathrm{NaCl}$ ) in topsoil. The biochar would preferably adsorb divalent cations (e.g., $\mathrm{Ca}^{2+}$ ) from fertilizers and groundwater than $\mathrm{Na}^{+}$, thus increasing the relative abundance of $\mathrm{Na}^{+}$in soil solution and producing a higher SAR value. $\mathrm{SO}_{4}^{2-}$ tends to combine with $\mathrm{Ca}^{2+}$ of soil particles and biochar to form a less soluble compound, indirectly increasing $\mathrm{Cl}^{-} / \sqrt{ } \mathrm{SO}_{4}^{2-}$ (Fig. 2h). Nevertheless, both SAR and $\mathrm{Cl}^{-} / \sqrt{ } \mathrm{SO}_{4}^{2-}$ of T4-T6 treatments at wheat and maize harvest times were lower than $\mathrm{CK}$ values, suggesting a conjunctive effect of biochar and $75 \% \mathrm{CF}$ on producing a less toxic soil solution for crop growth.

\subsection{Crop uptake of $\mathrm{Na}$ and $\mathrm{K}$ in response to biochar use}

The wheat season is drier than maize season in the YRD, and wheat is more vulnerable to salt stress. As shown in Fig. 3a, with the increase of biochar dose from 0 to 3,6 , and $12 \mathrm{tha}^{-1}$, the $\mathrm{K} / \mathrm{Na}$ ratio increased from 0.77 to $0.86,0.94$, and 0.95 , though their differences were not statistically significant due to the large variations among replicates. Coapplications of biochar $\left(3,6\right.$, and $12 \mathrm{t} \mathrm{ha}^{-1}$ ) and 75\% CF (T4-T6) significantly increased the $\mathrm{K} / \mathrm{Na}$ ratio over $75 \% \mathrm{CF}$. Mainly, T5 and T6 had higher $\mathrm{K} / \mathrm{Na}$ ratios than $\mathrm{CF}$ (Fig. 3b). The K-rich biochar enhanced

Table 6

$\mathrm{NH}_{4}^{+}-\mathrm{N}, \mathrm{NO}_{3}^{-}-\mathrm{N}$, Olsen-P and organic matter of the soil at crop harvests

\begin{tabular}{|c|c|c|c|c|c|c|c|c|}
\hline \multirow[b]{2}{*}{ Treatments } & \multicolumn{2}{|l|}{$\mathrm{NH}_{4}^{+}-\mathrm{N}$} & \multicolumn{2}{|l|}{$\mathrm{NO}_{3}^{-}-\mathrm{N}$} & \multicolumn{2}{|l|}{ Olsen-P } & \multicolumn{2}{|l|}{ Organic matter } \\
\hline & Wheat harvest & Maize harvest & $\begin{array}{l}\text { Wheat harvest } \\
\mathrm{ng} \mathrm{kg}^{-1} \text { ) }\end{array}$ & Maize harvest & Wheat harvest & Maize harvest & $\begin{array}{l}\text { Wheat harvest } \\
\left(\mathrm{g} \mathrm{kg}^{-1}\right)\end{array}$ & Maize harvest \\
\hline CK & $2.69 \pm 0.49 b$ & $4.18 \pm 0.06 a$ & $27.11 \pm 0.67 \mathrm{a}$ & $1.21 \pm 0.23 c$ & $0.59 \pm 0.02 c$ & $0.52 \pm 0.01 b$ & $6.18 \pm 0.17 c$ & $6.87 \pm 0.29 b$ \\
\hline $\mathrm{T} 1$ & $3.59 \pm 0.05 a$ & $2.17 \pm 0.09 c$ & $17.56 \pm 0.97 b$ & $1.07 \pm 0.08 c$ & $2.04 \pm 0.17 b$ & $0.73 \pm 0.06 b$ & $7.43 \pm 0.14 b c$ & $8.00 \pm 0.69 a b$ \\
\hline $\mathrm{T} 2$ & $4.20 \pm 0.24 a$ & $2.96 \pm 0.33 b$ & $13.87 \pm 0.10 c$ & $4.10 \pm 0.41 b$ & $1.99 \pm 0.07 b$ & $0.72 \pm 0.12 b$ & $8.29 \pm 0.91 b$ & $7.29 \pm 0.03 b$ \\
\hline T3 & $3.59 \pm 0.05 a$ & $3.94 \pm 0.04 a$ & $8.95 \pm 0.55 d$ & $5.87 \pm 0.25 a$ & $2.31 \pm 0.04 a$ & $3.29 \pm 0.13 a$ & $10.26 \pm 0.28 \mathrm{a}$ & $9.04 \pm 0.36 a$ \\
\hline $\mathrm{CF}$ & $4.88 \pm 0.78$ & $3.32 \pm 0.24$ & $34.42 \pm 6.72$ & $15.91 \pm 5.47$ & $3.10 \pm 0.81$ & $2.35 \pm 0.34$ & $7.28 \pm 0.04$ & $8.82 \pm 0.09$ \\
\hline $75 \%$ CF & $4.41 \pm 0.57 \mathrm{ab}$ & $2.78 \pm 0.21 \mathrm{a}$ & $28.60 \pm 1.33 \mathrm{a}$ & $17.87 \pm 1.62 b$ & $1.26 \pm 0.06 c$ & $1.49 \pm 0.03 a$ & $6.93 \pm 0.56 b$ & $7.73 \pm 0.50 b$ \\
\hline $\mathrm{T} 4$ & $3.03 \pm 0.71 \mathrm{ab}$ & $1.35 \pm 0.07 \mathrm{a}$ & $26.05 \pm 20.85 a$ & $5.54 \pm 1.50 \mathrm{~d}$ & $0.59 \pm 0.15 d$ & $0.57 \pm 0.01 \mathrm{a}$ & $7.48 \pm 0.54 a b$ & $6.91 \pm 0.35 b$ \\
\hline T5 & $4.83 \pm 0.72 \mathrm{ab}$ & $2.16 \pm 1.26 \mathrm{a}$ & $28.71 \pm 5.64 a$ & $10.58 \pm 0.75 c$ & $2.28 \pm 0.40 \mathrm{~b}$ & $0.77 \pm 0.30 \mathrm{a}$ & $9.14 \pm 0.61 \mathrm{a}$ & $7.59 \pm 0.02 b$ \\
\hline T6 & $7.85 \pm 2.80 \mathrm{a}$ & $3.07 \pm 0.01 \mathrm{a}$ & $15.41 \pm 9.48 \mathrm{a}$ & $23.04 \pm 0.98 a$ & $4.09 \pm 0.53 a$ & $1.97 \pm 1.15 \mathrm{a}$ & $8.79 \pm 0.73 a$ & $9.22 \pm 0.04 \mathrm{a}$ \\
\hline
\end{tabular}

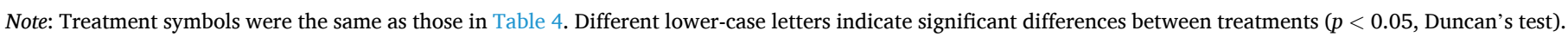



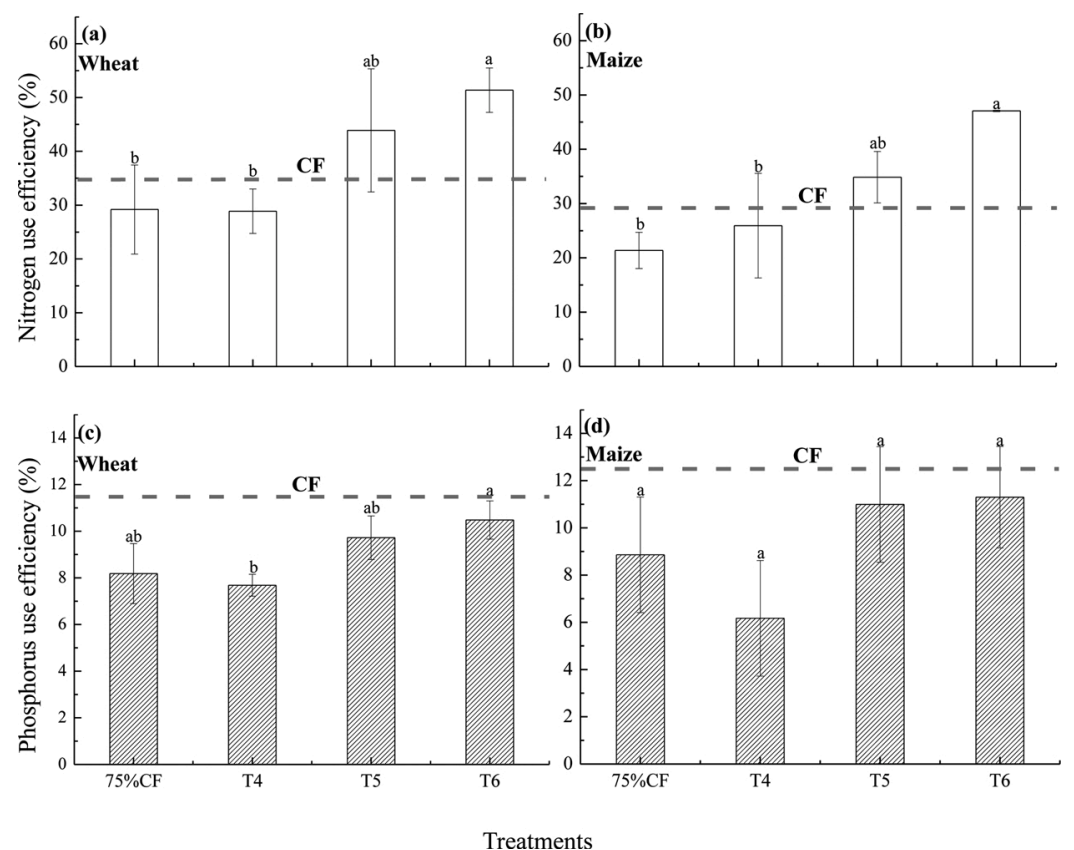

Fig. 4. Nitrogen (a, b) and phosphorus (c, d) use efficiency in response to the co-applications of biochar and fertilizers. Treatments were the same as those in Fig. 2. Different lower-case letters indicate significant differences between treatments $(p<0.05$, Duncan's test).

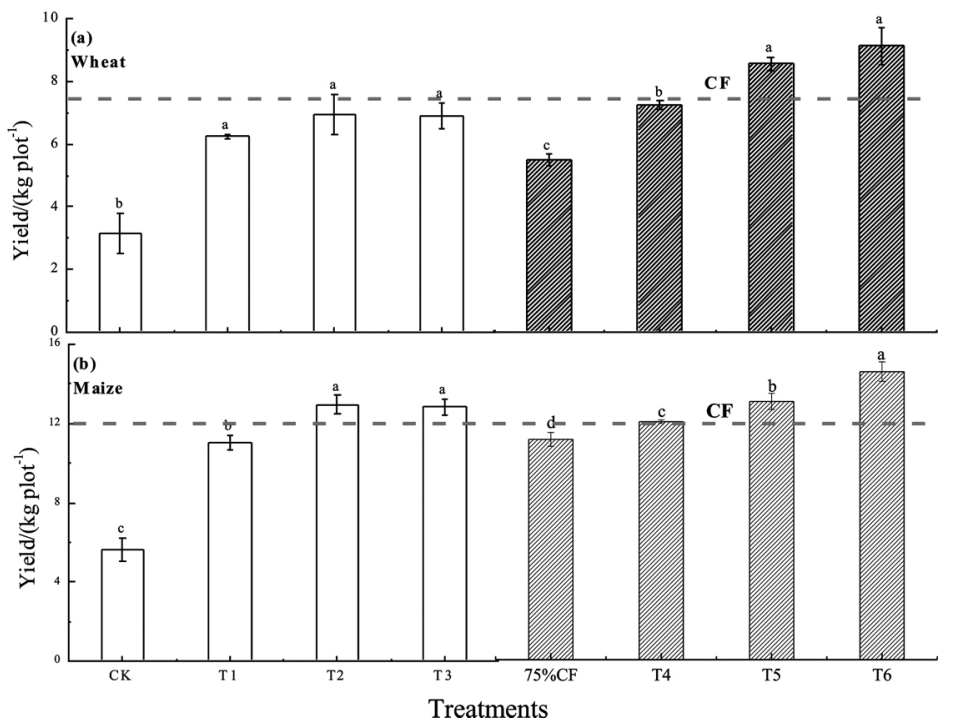

Fig. 5. Wheat (a) and maize (b) yields as affected by biochar alone or the co-applications of biochar and fertilizers. Treatments were the same as those in Fig. 2 . Different lower-case letters indicate significant differences between treatments $(p<0.05$, Duncan's test).

$\mathrm{K}^{+}$uptake by wheat, probably due to the changes in soil solution composition.

\subsection{Nutrient use efficiency in response to biochar use}

Biochar use alone (T1-T3) and in combination with fertilizers (T4-T6) affected soil nutrient and soil organic matter (SOM) contents at wheat and maize harvest times (Table 6). $\mathrm{NH}_{4}^{+}-\mathrm{N}$, Olsen-P, and SOM contents generally increased, whereas $\mathrm{NO}_{3}^{-}-\mathrm{N}$ content sharply decreased with biochar use (T1-T3) over CK at wheat harvest time. In contrast, at maize harvest time, neither T1-T3 nor T4-T6 had a positive effect on $\mathrm{NH}_{4}^{+}-\mathrm{N}$, whereas $\mathrm{T} 3$ or T6 markedly increased $\mathrm{NO}_{3}^{-}-\mathrm{N}$, Olsen-P, and SOM contents over $\mathrm{CK}$ or $75 \% \mathrm{CF}$.

T4-T6 treatments gradually increased the NUE with biochar doses (Fig. 4a, b). Notably, T5-T6 improved wheat NUE by $33.5-39.4 \%$ and maize NUE by $38.71 \%$ and $54.61 \%$ over $75 \%$ CF. Further, T5-T6 had higher NUE values than that of conventional fertilization (CF). PUE was slightly improved for wheat, but insignificant for maize. For both crops, 
PUE of biochar treatments was lower than CF.

\subsection{Crop yields in response to biochar use}

Biochar use alone (T1-T3) or in combination with 75\% CF (T4-T6) significantly increased crop yields over CK or 75\% CF (Fig. 5). T1-T3 increased wheat yield by $49.60 \%-54.68 \%$ and maize yield by $49.23 \%-$ $56.68 \%$. Indeed, at 6 and $12 \mathrm{tha}^{-1}$ doses, biochar alone resulted in wheat and maize yields similar to $\mathrm{CF}$, and biochar and $75 \% \mathrm{CF}$ produced yields higher than CF. In other words, reducing fertilizer use by $25 \%$ would not affect crop yields of the year if biochar was added at 6 or $12 \mathrm{tha}^{-1}$.

\section{Discussion}

\subsection{Biochar alleviates soil compaction and salt stress}

Adding biochar $\left(3,6\right.$, and $\left.12 \mathrm{tha}^{-1}\right)$ along with straw returning via rotary tillage to the salt-affected soil reduced its bulk density (BD) and increased its saturated hydraulic conductivity (Ks) (Fig. 1), which in turn helped remove soluble salts (Table 5) in soil upon rain and irrigation and reduce osmotic pressure of soil solution (Zörb et al., 2019). The large specific surface area $\left(27.5 \mathrm{~m}^{2} \mathrm{~g}^{-1}\right)$ and irregular shapes of the biochar could be crucial to the alleviation of soil compaction. Irregular and fluffy biochar particles would help soil particles form a porous structure, thus enhancing salt removal via irrigation (Liu et al., 2017; Baiamonte et al. 2019). The creation of a secondary pore system, such as macro-pores, might also play a role in salt leaching (Shaygan and Baumgartl, 2020). Further, the biochar had abundant $\mathrm{K}^{+}(683.4 \mathrm{mmol}$ $\left.\mathrm{kg}^{-1}\right), \mathrm{Ca}^{2+}\left(227.7 \mathrm{mmol} \mathrm{kg}{ }^{-1}\right), \mathrm{Mg}^{2+}\left(356.1 \mathrm{mmol} \mathrm{kg}^{-1}\right)$, and $\mathrm{SO}_{4}^{2-}$ $\left(112.9 \mathrm{mmol} \mathrm{kg}^{-1}\right)$, release of which would adjust salt compositions in the soil solution to more favorable conditions for crop growth.

The possible mechanisms by which biochar altered soil solution compositions could be summarized as (a) biochar released $\mathrm{K}^{+}, \mathrm{Ca}^{2+}$, and $\mathrm{Mg}^{2+}$ to exchange with $\mathrm{Na}^{+}$in soil solution thereby reducing $\mathrm{Na}^{+}$activity and toxicity (Lin et al., 2015; Zheng et al., 2018); and (b) the released $\mathrm{K}^{+}, \mathrm{Ca}^{2+}$, and $\mathrm{Mg}^{2+}$ to soil solution displaced $\mathrm{Na}^{+}$on the soil particles and helped $\mathrm{Na}^{+}$leaching with irrigation (Usman et al., 2016). The decrease in SAR and $\mathrm{Cl}^{-} / \sqrt{ } \mathrm{SO}_{4}^{2-}$ at wheat and maize harvest times (Fig. 2) provides evidence of the mechanisms. Co-applications of biochar with fertilizers, however, complicated the changes in SAR and $\mathrm{Cl}^{-} / \sqrt{ } \mathrm{SO}_{4}^{2-}$ for the $2^{\text {nd }}$ mechanism.

Reduced $\mathrm{Na}^{+}$(Table 5) and SAR (Fig. 2) from biochar use lowered soil pH (Table 5). This phenomenon was also reported by Chaganti et al. (2015) and Shaygan et al. (2017). Further, the enhanced decomposition of straw by biochar would produce organic acids and reduce soil $\mathrm{pH}$ (Xiao et al., 2020).

The changes in osmotic pressure, salt compositions, and $\mathrm{pH}$ altogether helped crop absorb $\mathrm{K}^{+}$and expel $\mathrm{Na}^{+}$(Lashari et al., 2015; Kim et al., 2016), resulting in an increased $\mathrm{K} / \mathrm{Na}$ ratio of grains (Fig. 3). This ratio indicates the relief of salt stress (Lin et al., 2015; Ali et al., 2017). Among the treatments, T5-T6 increased the ratio the most, suggesting their most significant impact on alleviating $\mathrm{Na}^{+}$toxicity.

\subsection{Effects of biochar use on nutrient retention and supply}

With abundant carboxyl and phenolic-OH groups (Table 1), the biochar would enhance the retention of $\mathrm{NH}_{4}^{+}-\mathrm{N}$ in soil (Zheng et al., 2010; Al-Wabel et al., 2018). In contrast, at the $\mathrm{pH}$ of the soil (8.2), the carboxyl groups would be negatively charged, which was unfavorable to the retention of $\mathrm{NO}_{3}^{-} \mathrm{N}$ (Kameyama et al., 2012) at wheat harvest (Table 6). As biochar aged to the maize harvest time, $\mathrm{NO}_{3}^{-}-\mathrm{N}$ retention in soil increased with biochar dose, which may be explained by the higher contents of an organic coating (Hagemann et al., 2017), oxidized functional groups, and anion exchange capacity of aged biochar than a fresh one (Mia et al., 2017).

T1-T3 increased Olsen-P because the biochar had a much higher
Olsen-P content than the soil (Lashari et al., 2013). Besides reducing soil $\mathrm{pH}$ (Table 5), T3 could contribute to the release of available $\mathrm{P}$ by reducing the formation of Ca-P crystal phases (Saifullah et al., 2018).

Soils in the YRD are generally rich in potassium (Dong et al., 2006), resulting in high $\mathrm{K}^{+}$content in local reed and its derived biochar. Thus, the biochar can provide $\mathrm{K}^{+}$to the soil and enhance $\mathrm{K}^{+}$uptake by crops. Similar views were reported by Akhtar et al. (2015) and Lin et al. (2015).

Biochar use increased soil organic matter content (Table 6) via the indirect effect of biochar accelerating straw decomposition as a result of enhanced porosity and aeration in biochar-amended saline soil, as suggested by Xiao et al. (2020). T4-T6 treatments increased NUE, which was the combined result of nutrient inputs from biochar (Table 2), the enhanced nutrient retention by the functional groups of biochar (Table 6), and the increased SOM content (Table 6). SOM would enhance microbial activities and increase nutrient supply when crops need (Arif et al., 2017; Al-Wabel et al., 2018; Li et al., 2019).

\subsection{Effects of biochar and fertilizer use on crop yields}

Crop yields increased in the order of CK $<75 \% \mathrm{CF}<\mathrm{CF}$ (Fig. 5) explains the importance of fertilization in crop production. In contrast, crop yields of biochar treatment alone (T1-T3) were above that of $75 \%$ $\mathrm{CF}$ and close to $\mathrm{CF}$, suggesting that soil physical conditions could be more important than nutrient supply and retention to crop growth in one wheat-maize rotation (Fig. 5).

T5-T6 treatments further enhanced crop yields by alleviating salt stress (Fig. 3a, b) and improving NUE (Fig. 4a, b). This beneficial effect of biochar on crop yield may be attributed to 1) the reduced salt stress (the increase in $\mathrm{K} / \mathrm{Na}$ ) as a result of improved soil physical conditions and enhanced salt leaching, as discussed above; 2) the increased SOM content and nutrient (, P) availability; and 3) the provision of $\mathrm{K}$ nutrient from the biochar to crop growth (Lin et al., 2015). The results from this field trial indicate that alleviating soil compaction to enhance salt leaching is fundamental to crop production in saline soil in the YRD, and biochar is a useful soil amendment to achieve the objective.

Due to external factors beyond control, the field trial only lasted a year. It would be great if the observed multiple benefits of biochar improving soil physical properties, enhancing salt leaching, increasing nutrient use efficiency, and raising crop yields could be examined for multiple years in the YRD.

\section{Conclusions}

Inexpensive biochar produced in the field from local reed was trialed to remediate a compact soil with $2.8 \%$ salt. A single addition of biochar at 3,6 , and $12 \mathrm{tha}^{-1}$ doses (T1-T3) to a saline soil with straw returning by rotary tillage before wheat sowing alleviated soil compaction (i.e., soil BD decreased, and $K s$ increased) and produced a more favorable soil solution composition (SAR) for wheat and maize growth. The reduced contents of soluble salts (particularly the harmful $\mathrm{Na}^{+}$and $\mathrm{Cl}^{-}$) in soil solution helped alleviate salt stress, thus benefiting crop growth. Biochar also helped SOM accumulation and nutrient retention. Application of 6 and $12 \mathrm{t} \mathrm{ha}^{-1}$ biochar (T5-T6) in the field trial can achieve the goals of reducing the use of fertilizers (urea-ammonium mixed nitrogen fertilizer and slow-release fertilizer for wheat, and ammonium dihydrogen phosphate and urea for maize) by $25 \%$ and still improving crop yields in wheat-maize rotation.

\section{CRediT authorship contribution statement}

Liang Xiao: Investigation, Data curation, Writing - original draft. Guodong Yuan: Writing - review \& editing. Lirong Feng: Formal analysis. Dongxue Bi: Supervision. Jing Wei: Validation. 
Declaration of Competing Interest

Authors declare that no conflict of interest exists in the submission of this manuscript.

\section{Acknowledgments}

This work was supported by grants from the Chinese National Key Research and Development Program (2016YFD0200303) and Key Research and Development Program of Shandong Province (2016CYJS05A01). Two anonymous reviewers and editor are gratefully appreciated for their constructive comments and suggestions that improved this manuscript.

\section{References}

Ajayi, A.E., Holthusen, D., Horn, R., 2016. Changes in microstructural behavior and hydraulic functions of biochar amended soils. Soil Tillage Res. 155, 166-175.

Akhtar, S.S., Andersen, M.N., Liu, F., 2015. Residual effects of biochar on improving growth, physiology and yield of wheat under salt stress. Agric. Water Manage. 158, 61-68.

Ali, S., Rizwan, M., Qayyum, M.F., Ok, Y.S., Ibrahim, M., Riaz, M., Arif, M.S., Hafeez, F., Al-Wabel, M.I., Shahzad, A.N., 2017. Biochar soil amendment on alleviation of drought and salt stress in plants: a critical review. Environ. Sci. Pollut. Res. 24 (14), $12700-12712$.

Alvarez-Campos, O., Lang, T.A., Bhadha, J.H., McCray, J.M., Glaz, B., Daroub, S.H., 2018. Biochar and mill ash improve yields of sugarcane on a sand soil in Florida. Agric. Ecosyst. Environ. 253, 122-130.

Al-Wabel, M.I., Hussain, Q., Usman, A.R.A., Ahmad, M., Abduljabbar, A., Sallam, Abdulazeem, S., Ok, Y.S., 2018. Impact of biochar properties on soil conditions and agricultural sustainability: a review. Land Degrad. Dev. 29 (7), 2124-2161.

Arif, M., Ilyas, M., Riaz, M., Ali, K., Shah, K., Haq, I.U., Fahad, S., 2017. Biochar improves phosphorus use efficiency of organic-inorganic fertilizers, maize-wheat productivity and soil quality in a low fertility alkaline soil. Field Crops Res. 214, 25-37.

Baiamonte, G., Crescimanno, G., Parrino, F., Pasquale, C.D., 2019. Effect of biochar on the physical and structural properties of a desert sandy soil. Catena 175, 294-303.

Bao, S.D., 2000. Soil agro-chemical analysis, 3rd ed. China Agriculture Press, Beijing, pp. 187-196 (in Chinese).

Blanco-Canqui, H., 2017. Biochar and soil physical properties. Soil Sci. Soc. Am. J. 81, 687-711.

Burrell, L.D., Zehetner, F., Rampazzo, N., Wimmer, B., Soja, G., 2016. Long-term effects of biochar on soil physical properties. Geoderma 282, 96-102.

Chaganti, V.N., Crohn, D.M., Šimůnek, J., 2015. Leaching and reclamation of a biochar and compost amended saline-sodic soil with moderate SAR reclaimed water. Agric. Water Manage. 158, 255-265.

Di Lonardo, S., Baronti, S., Vaccari, F.P., Albanese, L., Battista, P., Miglietta, F., Bacci, L., 2017. Biochar-based nursery substrates: the effect of peat substitution on reduced salinity. Urban For. Urban Greening 23, 27-34.

Dong, H.Z., Xin, C.S., Tang, W., Li, W.J., Zhang, D.M., Wen, S.M., 2006. Seasonal changes of salinity and nutrients in the coastal saline soil in Dongying, Shandong, and their effects on cotton yield. Cotton Sci. 18 (6), 362-366 (in Chinese, with English abstract).

Gu, Y.F., Zhang, T., Che, H., Lu, X.Y., Du, Y.Q., 2015. Influence of returning corn straw to soil on soil nematode communities in winter wheat. Acta Ecol. Sin. 35 (2), 52-56 (in Chinese, with English abstract).

Hagemann, N., Joseph, S., Schmidt, H., Kammann, C., Harter, J., Borch, T., Young, R.B., Varga, K., Taherymoosav, S., Elliott, K.W., McKenna, A., Albu, M., Mayrhofer, C., Obst, M., Conte, P., Dieguez-Alonso, A., Orsetti, S., Subdiaga, E., Behrens, S., Kappler, A., 2017. Organic coating on biochar explains its nutrient retention and stimulation of soil fertility. Nat. Commun. 8 (1), 1089-1100.

International Humic Substance Society, 2019. 12.31. Acidic functional groups of IHSS samples accessed on December 31, 2019. http://humic-substances.org/acidic-funct ional-groups-of-ihss-samples/.

Kameyama, K., Miyamoto, T., Shiono, T., Shinogi, Y., 2012. Influence of sugarcane bagasse derived biochar application on nitrate leaching in calcaric dark red soil. J. Environ. Qual. 41 (4), 1131-1137.

Kim, H.S., Kim, K.R., Yang, J.E., Ok, Y.S., Owens, G., Nehls, T., Wessolek, G., Kim, K.H., 2016. Effect of biochar on reclaimed tidal land soil properties and maize (Zea mays L.) response. Chemosphere 142, 153-159.

Lashari, M.S., Liu, Y., Li, L., Pan, W., Fu, J., Pan, G., Zheng, J., Zheng, J., Zhang, X., Yu, X., 2013. Effects of amendment of biochar-manure compost in conjunction with pyroligneous solution on soil quality and wheat yield of a salt-stressed cropland from Central China Great Plain. Field Crop Res. 144, 113-118.

Lashari, M.S., Ye, Y.X., Ji, H.S., Li, L.Q., Kibue, G.W., Lu, H.F., Zheng, J.F., Pan, G.X., 2015. Biochar-manure compost in conjunction with pyroligneous solution alleviated salt stress and improved leaf bioactivity of maize in a saline soil from central China: a 2-year field experiment. J. Sci. Food Agric. 95, 1321-1327.

Lehmann, J., Gaunt, J., Rondon, M., 2006. Biochar sequestration in terrestrial ecosystems-a review. Mitigation Adapt. Strategies Global Change 11, 395-419.

Lesch, S.M., Suarez, D.L., 2009. Technical note: a short note on calculating the adjusted SAR index. Transactions of the ASABE. 52 (2), 493-496.
Li, S.L., Wang, S., Shangguan, Z.P., 2019. Combined biochar and nitrogen fertilization at appropriate rates could balance the leaching and availability of soil inorganic nitrogen. Agric. Ecosyst. Environ. 276, 21-30.

Lin, X.W., Xie, Z.B., Zheng, J.Y., Liu, Q., Bei, Q.C., Zhu, J.G., 2015. Effects of biochar application on greenhouse gas emissions, carbon sequestration and crop growth in coastal saline soil. Eur. J. Soil Sci. 66, 329-338.

Liu, Z.L., Dugan, B., Masiello, C.A., Gonnermann, H.M., 2017. Biochar particle size, shape, and porosity act together to influence soil water properties. PLoS One 12 (6), e0179079.

Lu, R.K., 1999. Analytical methods for soil and agricultural chemistry. China Agricultural Science and Technology Press, Beijing, pp. 107-108, 156-160, 180-182, 269-271, 306-316, 352-355, 362-366. (in Chinese).

Lu, X.S., Yu, D.S., Xu, Z.C., Huang, J.J., Zhou, C.C., Sun, B., 2019. Study on comprehensive quantitative relationship of soil fertility quality and nitrogen application rate with wheat nitrogen use efficiency. Acta Pedologica Sin. 56 (2) 487-494 (in Chinese, with English abstract).

Luo, X.X., Liu, G.C., Xia, Y., Chen, L., Jiang, Z.X., Zheng, H., Wang, Z.Y., 2017. Use of biochar-compost to improve properties and productivity of the degraded coastal soil in the Yellow River Delta. China. J. Soils Sediments 17 (3), 780-789.

Mia, S., Singh, B., Dijkstra, F.A., 2017. Aged biochar affects gross nitrogen mineralization and recovery: a 15N study in two contrasting soils. GCB Bioenergy 9 (7), 1196-1206.

Nguyen, T.T.N., Xu, C.Y., Tahmasbian, I., Che, R.X., Xu, Z.H., Zhou, X.H., Wallace, H.M., Bai, S.H., 2017. Effects of biochar on soil available inorganic nitrogen: a review and meta-analysis. Geoderma 288, 79-96.

Obia, A., Mulder, J., Martinsen, V., Cornelissen, G., Børresen, T., 2016. In situ effects of biochar on aggregation, water retention and porosity in light-textured tropical soils. Soil Tillage Res. 155, 35-44.

Saifullah, Dahlawi, S., Naeem, A., Rengel, Z., Naidu, R., 2018. Biochar application for the remediation of salt-affected soils: challenges and opportunities. Sci. Total Environ. 625, 320-335.

Sastre-Conde, I., Lobo, M.C., Beltrán-Hernández, R.I., Poggi-Varaldo, H.M., 2015. Remediation of saline soils by a two-step process: washing and amendment with sludge. Geoderma 247-248, 140-150.

Shan, J.J., Chen, X.B., Yin, C.Y., Wen, P., Yan, K., Zhang, L.H., Zhang, L.B., Fu, X.Q., Sun, H.S., 2017. Comparison of fertilizer-effect models on winter wheat response to nitrogen and phosphorus fertilizers in saline soils in the Yellow River Delta. Chin. J. Eco-Agric. 25 (7), 1016-1024 (in Chinese with English abstract).

Shaygan, M., Reading, L.P., Baumgartl, T., 2017. Effect of physical amendments on salt leaching characteristics for reclamation. Geoderma 292, 96-110.

Shaygan, M., Baumgartl, T., 2020. Simulation of the effect of climate variability on reclamation success of brine-affected soil in semi-arid environments. Sustainability 12, 371-394.

Shao, M.A., Wang, Q.J., Huang, M.B., 2006. Soil physics. Higher Education Press, Beijing, pp. 37-38, 84. (in Chinese).

Sun, H.J., Lu, H.Y., Chu, L., Shao, H.B., Shi, W.M., 2017. Biochar applied with appropriate rates can reduce $\mathrm{N}$ leaching, keep $\mathrm{N}$ retention and not increase $\mathrm{NH}_{3}$ volatilization in a coastal saline soil. Sci. Total Environ. 575, 820-825.

Sun, H.J., Zhang, H.C., Shi, W.M., Zhou, M.Y., Ma, X.F., 2019. Effect of biochar on nitrogen use efficiency, grain yield and amino acid content of wheat cultivated on saline soil. Plant Soil Manage. 65 (2), 83-89.

Tedeschi, A., Lavini, A., Riccardi, M., Pulvento, C., D' Andria, R., 2011. Meloncrops (Cucumis melo L., cv. Tendral) grown in a Mediterranean environment under salinesodic conditions: part I. Yield and quality. Agric. Water Manage. 98 (9), 1339-1348.

Usman, A.R., Al-Wabel, M.I., Abdulaziz, A.H., Mahmoud, W.A., EL-Naggar, A.H., Ahmad, M., Abdulelah, A.F., Abdulrasoul, A.O., 2016. Conocarpus biochar induces changes in soil nutrient availability and tomato growth under saline irrigation. Pedosphere 26, 27-38.

Vochozka, M., Marouskova, A., Vachal, J., Strakova, J., 2016. Biochar pricing hampers biochar farming. Clean Technol. Environ. Policy 18 (4), 1225-1231.

Wang, J., Xiao, L., Bi, D.X., Wei, J., Yuan, G.D., 2018. Processes of leonardite altering cation and anion composition of soil solution in salt-affected soil in the Yellow River Delta. Acta Pedologica Sin. 55 (6), 1367-1376 (in Chinese, with English abstract).

Wong, V., Dalal, R., Greene, R., 2009. Carbon dynamics of sodic and saline soils following gypsum and organic material additions: a laboratory incubation. Appl. Soil Ecol. 41 (1), 29-40.

Wu, C.W., Chen, X.B., Shan, J.J., Yin, C.Y., Zhang, L.B., 2016. Comprehensive evaluation of crop straw resources in the Yellow River Delta: a case study of Dongying. J. Ecol. Rural Environ. 32 (3), 512-516 (in Chinese with English abstract).

Xiao, L., Yuan, G.D., Bi, D.X., Wei, J., Shen, G.H., 2019. Equipment and technology of field preparation of biochars from agricultural and forest residues under aerobic conditions with water-fire coupled method. Trans. Chin. Soc. Agric. Eng. 35 (11), 239-244 (in Chinese, with English abstract).

Xiao, L., Yuan, G.D., Feng, L.R., Bi, D.X., Wei, J., Shen, G.H., Liu, Z.H., 2020. Coupled effects of biochar use and farming practice on physical properties of a salt-affected soil with wheat-maize rotation. J. Soils Sediments. https://doi.org/10.1007/s11368020-02616-0.

Xie, W.J., Wu, L.F., Zhang, Y.P., Wu, T., Li, X.P., Ouyang, Z., 2017. Effects of straw application on coastal saline topsoil salinity and wheat yield trend. Soil Tillage Res. $169,1-6$.

Yang, L., Bian, X., Yang, R., Zhou, C., Tang, B., 2018. Assessment of organic amendments for improving coastal saline soil. Land Degrad. Dev. 29 (9), 3204-3211.

Yu, H.W., Zou, W.X., Chen, J.J., Chen, H., Yu, Z.B., Huang, J., Tang, H.R., Wei, X.Y., Gao, B., 2019. Biochar amendment improves crop production in problem soils: a review. J. Environ. Manage. 232, 8-21. 
Zhang, T., Wang, T., Liu, K.S., Wang, L.X., Wang, K., Zhou, Y., 2015. Effects of different amendments for the reclamation of coastal saline soil on soil nutrient dynamics and electrical conductivity responses. Agr. Water Manage. 159, 115-122.

Zheng, H., Wang, X., Chen, L., Wang, Z., Xia, Y., Zhang, Y., Wang, H., Luo, X., Xing, B., 2018. Enhanced growth of halophyte plants in biochar-amended coastal soil: roles of nutrient availability and rhizosphere microbial modulation. Plant Cell Environ. 41 (3), 517-532.

Zörb, C., Geilfus, C.M., Dietz, K.J., 2019. Salinity and crop yield. Plant Biol. 21, 31-38. 